\title{
Agôn
}

Revue des arts de la scène

HS 2 | 2014

Mettre en scène le conte

\section{Cendrillon de Joël Pommerat : Le conte sous le palimpseste}

\section{Christiane Connan-Pintado}

\section{(2) OpenEdition}

Journals

\section{Édition électronique}

URL : http://journals.openedition.org/agon/3146

DOI : $10.4000 /$ agon.3146

ISSN : 1961-8581

Éditeur

Association Agôn

\section{Référence électronique}

Christiane Connan-Pintado, « Cendrillon de Joël Pommerat : Le conte sous le palimpseste », Agôn [En ligne], HS 2 | 2014, mis en ligne le 04 mars 2015, consulté le 03 mai 2019. URL : http:// journals.openedition.org/agon/3146; DOI : 10.4000/agon.3146

Ce document a été généré automatiquement le 3 mai 2019.

Association Agôn et les auteurs des articles 


\title{
Cendrillon de Joël Pommerat : Le conte sous le palimpseste
}

\author{
Christiane Connan-Pintado
}

1 Les travaux des anthropologues ${ }^{1}$ ont mis en évidence la présence du conte «Cendrillon » dans différentes aires géoculturelles, et l'on sait qu'il figure, avec « Le Petit Poucet » et «La Barbe bleue », dans le palmarès des contes de Perrault les plus fréquemment soumis à la variation dramatique ${ }^{2}$. Pour nous inscrire dans ce dossier consacré à la transposition théâtrale du conte à travers l'exemple du spectacle de Joël Pommerat, nous ne reviendrons pas sur cette mise en perspective historique, rappelée par Marie Bernanoce ${ }^{3}$ qui, en spécialiste du théâtre pour la jeunesse, se consacre à l'analyse dramaturgique de la pièce. Nous choisissons pour notre part de l'aborder autrement, au prisme de notre intérêt pour les réécritures de contes, qui nous conduit à interroger la présence vivante de(s) contes(s) source(s) sous le palimpseste contemporain.

\section{Retour aux contes sources}

2 Il convient en effet d'évoquer ces contes sources au pluriel car on peut percevoir les influences conjuguées de Perrault et des Grimm sur le Cendrillon de Joël Pommerat. Pourtant, tout en s'inscrivant dans le cadre du même conte type (AT $510 \mathrm{~A}$ d'après la classification internationale), les deux versions apparaissent sensiblement différentes, comme le souligne Jean Bellemin-Noël, lors de son approche «textanalytique ${ }^{4}$ » des contes :

Notre "Cendrillon" est un autre conte que "Aschenputtel" des Grimm. Perrault a pour ainsi dire châtré la castration. [...] Il n'empêche que petits Français et petits Allemands lisent sous le même nom de "Cendrillon" deux contes fort différents, particulièrement quant à leurs résonances affectives et, sans doute, inconscientes ${ }^{5}$. Nettement moins présent dans l'édition française, le conte allemand y fait rarement l'objet de réécritures. Sans doute éditeurs et auteurs préfèrent-ils opter pour la version du patrimoine national. Aussi ignore-t-on le plus souvent en France que nulle marraine n'est convoquée chez les Grimm, partant nulle citrouille avec son pittoresque équipage de rats et de lézards. 
Deux fois plus long que celui de Perrault, le conte des Grimm étoffe chacune de ses étapes et y insère de nouveaux épisodes : au lieu de s'ouvrir sur le remariage du père, il détaille le récit de la mort de la première épouse, ses dernières paroles et les visites de Cendrillon au cimetière; partant un jour en voyage, le père interroge les trois jeunes filles sur le cadeau qu'elles aimeraient recevoir à son retour et Cendrillon demande un simple rameau alors que ses sœurs réclament toilettes et bijoux ${ }^{6}$; planté sur la tombe de sa mère, arrosé de ses larmes, le rameau devient un noisetier bruissant d'oiseaux, futurs adjuvants de la jeune fille tout au long du conte ; ainsi, lorsque pour l'empêcher d'aller au bal, sa belle-mère lui impose des épreuves insurmontables comme de trier des lentilles jetées dans la cendre, les oiseaux accomplissent la tâche; de plus, le conte des Grimm est jalonné et agrémenté de formulettes chantées par Cendrillon et adressées à l'arbre, ou par les oiseaux commentant l'action; ce sont eux qui lui fournissent sa tenue pour le bal, auquel elle se rend à trois reprises - contre une seule chez Perrault ; la troisième fois, le prince fait enduire de poix l'escalier, et par cette ruse retient la pantoufle d'or - et non de verre - grâce à laquelle il identifie la belle inconnue. Par-delà ces différences de structure ou de détail, le lecteur est avant tout frappé par l'extrême cruauté avec laquelle sont traitées les sœurs de Cendrillon. On se souvient que, dans le conte de Perrault, elle sont finalement pardonnées, logées au palais et mariées à des seigneurs de la cour. En revanche, les Grimm décrivent les mutilations qu'elles s'infligent, sur les conseils de leur mère, pour essayer la petite pantoufle : l'une se tranche l'orteil et l'autre le talon tandis que les oiseaux attirent l'attention du prince sur le pied sanglant des usurpatrices; au dénouement, les tendres colombes qui avaient soutenu Cendrillon crèvent les yeux des deux sœurs, supplice détaillé à plaisir, un œil chacune en entrant dans l'église lors du mariage, l'autre à la sortie. Loin de l'ironie enjouée du conteur français et de ses moralités équivoques, l'explicit des Grimm se situe entièrement du côté du conte d'avertissement : «Et la cécité fut donc la punition de leur méchanceté et de leur perfidie pour le restant de leurs jours ${ }^{7} »$.

4 Si Perrault veille à ménager les bienséances, les motifs réunis dans le conte allemand reflètent davantage le répertoire populaire, sa rusticité et sa cruautés. Les deux versions ne sauraient se confondre et celle des Grimm n'étant guère connue en France, Bruno Castan fait figure d'exception lorsqu'il revendique son parrainage pour sa propre transposition théâtrale, La Fille aux oiseaux. Il s'en explique dans une postface où, se démarquant de l'auteur français, il dit sa fascination pour les choix des frères Grimm :

La baguette magique de la fée, la citrouille-carrosse et ses valets-petits-rats ne me passionnent pas outre mesure chez Charles [...].

Et merci encore aux frères Grimm (et à leurs sources) pour la branche qui fouette le père, pour le talon et l'orteil tranchés, pour les yeux des harpies crevés par le bec rose des blanches colombes... Merci pour le sang...

Merci pour la cruauté...

Merci pour les oiseaux...

Merci pour la passion ${ }^{9} .$.

Castan se distingue par-là des réécritures de "Cendrillon» qui font couramment allégeance à l'hypotexte français et dans lesquelles le motif merveilleux de la citrouille changée en carrosse apparaît comme axe narratif majeur, qu'il s'agisse de pochades ${ }^{10}$, d'œuvres légitimées ${ }^{11}$ ou du long métrage d'animation de Disney en 1950. Moins dérangeante, la version française a prévalu jusque dans le monde anglo-saxon comme en atteste le travail du plasticien William Wegman ${ }^{12}$ qui photographie des chiens mis en scène dans des situations et des postures anthropomorphisées: l'un d'eux porte le 
costume et la baguette de la fée-marraine et un autre, en perruque blonde et bouclée, se rend au bal dans un carrosse orange et arrondi en forme de citrouille.

Les transpositions théâtrales de «Cendrillon » que nous avons pu repérer ne se soucient guère de référer à une source précise et semblent se fonder simplement sur la trame du conte conservée en mémoire. Suzanne Rominger publie un Cendrillon dépoussiérée ${ }^{13}$ parodique et marqué par la version de Disney, dans une collection à visée pédagogique, assortie de quelques conseils de jeu et de mise en scène destinés à un spectacle d'enfants. La pièce de René Pillot Cendres... Cendrillon ${ }^{14}$ s'inscrit dans le contexte géographique et social du Nord, région d'origine de l'auteur : à la fois réaliste et poétique, elle donne à voir l'affrontement des classes sociales en pays minier à la fin du XIX ${ }^{\mathrm{e}}$ siècle. D'autre part, quittant le domaine de la littérature de jeunesse, on signalera le «dramolet » de Robert Walser, œuvre de jeunesse publiée en 1901, qui a fait récemment l'objet d'une nouvelle traduction ${ }^{15}$. L'auteur allemand, qui a aussi réécrit «Blanche-Neige » et « La Belle au bois dormant ", s'inspire de la version Grimm, sans que rien dans sa réécriture poétique épurée ne l'indique précisément. Pour Pascale Auraix-Jonchière,

[...] les jeux de la récriture, qui deviennent eux-mêmes objets de l'écriture, ont une seconde finalité : transformer le dit du conte en matière poétique. Le canevas narratif oscille et se défait, le statut des personnages vacille, le texte de référence lui-même s'estompe, sujet au brouillage et support de greffes multiples, mais corrélativement un processus de poétisation est à l'œuvre ${ }^{16}$.

Dans cette version versifiée et métathéâtrale, au service d'une célébration de l'imagination et de la rêverie, on notera la présence d'un personnage inattendu, «le conte, habillé fantastiquement » (p. 29), qui surgit au cœur de la pièce où il fait un bref passage, en quelques répliques, parlant de lui à la troisième personne, pour conseiller Cendrillon, avant de « retourne[r] vers / sa fin, là- bas, d'où il vient » (p. 32).

\section{Relecture et recréation de « Cendrillon » par Joël Pommerat}

6 Joël Pommerat relate dans plusieurs entretiens ${ }^{17}$ comment, après ses deux pièces pour enfants inspirées de contes, Le Petit Chaperon rouge et Pinocchio ${ }^{18}$, il en est venu à s'intéresser à «Cendrillon». Alors qu'il n'avait gardé en mémoire que quelques traces issues des versions de Perrault et de Disney, la relecture du conte des Grimm agit comme un déclencheur lorsqu'il prend conscience du rôle essentiel joué dans l'économie du conte par le deuil inaugural, à la mort de la mère. Toute sa propre création s'organise dès lors à partir de cette thématique et le poids de la version Grimm joue un rôle évident dans son inspiration. Comme dans le conte allemand, Pommerat ménage un lien constant entre mère et fille, même si cette communication ne s'effectue plus par l'intermédiaire du noisetier et des oiseaux adjuvants, mais à travers la tentative désespérée de Cendrillon pour maintenir intact le souvenir de sa mère. Cependant, on peut noter que cet engagement est scellé au début de la pièce par un serment qui fait nettement écho aux oiseaux des Grimm :

Maman, je te promets que je penserai à toi à chaque instant. J'ai très bien compris que c'est grâce à ça que tu ne mourras pas en vrai et que tu resteras en vie dans un endroit secret invisible tenu par des oiseau $x^{19}$.

Métaphore de la tombe, cet « endroit secret invisible tenu par des oiseaux » réintroduit le merveilleux du conte dans un épisode que la transposition théâtrale traite sur un mode plus réaliste. De plus, le motif des oiseaux est reconfiguré de façon spectaculaire à travers 
l'évocation récurrente des oiseaux pris au piège de la maison de verre de la belle-mère ils viennent s'écraser contre ses parois et leurs cadavres jonchent le sol. Le motif souligne le clivage entre les deux figures maternelles, l'une en harmonie avec le monde des oiseaux, l'autre causant leur perte. D'autres allusions distillées au fil de la pièce convoquent le conte allemand, comme l'exclamation de la sœur aînée lors des préparatifs pour le bal: «En tout cas, moi je me ferais couper un pied pour pouvoir voir le prince avant tous les autres. Vous vous rendez pas compte, c'est grandiose ${ }^{20} »$. Apparemment gratuite, dans sa tournure hyperbolique, l'allusion qui pourrait être prémonitoire se concrétise dans les répliques suivantes de la sœur aînée : «A mort » et «Je meurs si j’y pense trop ", illustrant à l'occasion d'un épisode annexe ce poids des mots qui est bien le sujet profond de la pièce.

La prééminence de la source allemande n'a pas effacé l'influence du conte de Perrault, auquel Pommerat emprunte les jeux sur l'onomastique et le personnage de la fée. Lorsque les différents personnages de la pièce s'adressent à celle que le texte didascalique désigne comme "la très jeune fille», la déclinaison des noms Sandra, Sandrine, Cendrier et Cendrillon reprend et amplifie sur le mode parodique ce choix des surnoms-portraits dégradants que l'on trouvait déjà chez Perrault :

Lorsqu'elle avait fait son ouvrage, elle s'allait mettre au coin de la cheminée, et s'asseoir dans les cendres, ce qui fait qu'on l'appelait communément dans le logis Cucendron. La cadette, qui n'était pas si malhonnête que son aînée, l'appelait Cendrillon ${ }^{21}$.

Qu'il se réclame plutôt des Grimm ne conduit pas pour autant Pommerat à renoncer au personnage de la fée, riche en potentialités comiques. Ici encore il s'inscrit dans le sillage de Perrault qui s'écarte des usages en cours, lors de la mode des contes sous l'Ancien Régime, et traite lui-même avec désinvolture cette figure du personnel merveilleux. En effet les sortilèges mis en œuvre par les fées de ses contes sont moins extravagants que ceux que l'on trouve dans les contes de Madame d'Aulnoy ou de Madame Leprince de Beaumont. Comme l'a montré Marc Escola :

Au sein de la narration enjouée, la féerie est moins affaire de fiction que de diction : tout est mis en œuvre pour favoriser à l'égard du merveilleux féerique lui-même une prise de distance à la fois complice et amusée ${ }^{22}$.

La marraine-fée de Perrault se montre capable d'équiper sa filleule pour aller au bal, mais il lui faut prendre appui sur le quotidien le plus trivial pour opérer des métamorphoses; de plus son pouvoir ne s'exerce que pour un temps limité : à minuit, tout reprendra sa forme originelle. Pommerat accentue cette inaptitude du personnage magique à jouer son rôle et dynamite ainsi la féerie : chacune des interventions de la fée donne lieu à des échanges comiques, salutaire contrepoint à la gravité de la pièce.

Relèvent de la même veine parodique tous les détournements d'épisodes, comme celui de la fameuse pantoufle, présente dans le titre du conte de Perrault, mais ici redoublée et dégradée par l'inversion parodique: devant le château, la belle-mère "perd une chaussure, un des gardes la ramasse. Elle s'enfuit en boitant » (p. 98); plus loin, le jeune prince donne l'une de ses propres chaussures à Cendrillon en souvenir (p. 103), chaussure qui servira à sa reconnaissance par le roi à la scène suivante :

LE ROI (examinant la chaussure). Attendez voir... Ben si, c'est la chaussure de mon fils, c'est marqué le nom du fabricant à l'intérieur ! (p. 108)

Par-delà les sources et les jeux intertextuels, c'est de manière plus générale dans la lignée du conte comme forme narrative que s'inscrit Pommerat, à travers le choix déjà éprouvé, dans ses pièces antérieures, de l'épicisation. Après «L'homme qui raconte » du Petit 
Chaperon rouge, c'est «La voix de la narratrice » qui ouvre, clôt et ponctue les étapes de Cendrillon, et qui témoigne de la prégnance de cette pulsion conteuse. Le théâtre de Pommerat montre et conte à la fois, comme s'il ne pouvait reprendre les contes du patrimoine sans faire intervenir sur scène la figure ou la voix du conteur. Une voix adressée, qui lie et crée du lien, voix chargée d'une force illocutoire, interpellant le lecteur, commentant l'action, questionnant, résumant, anticipant, supervisant l'amont et l'aval de la fiction. Outre la présence de cette voix narratrice, force est de constater la récurrence du terme "histoire » et des syntagmes qu'il compose souvent avec le verbe « raconter » tout au long de la pièce. De l'incipit au dénouement, il s'agit toujours de raconter des histoires ou, plus risqué, de se raconter des histoires, comme le montre le florilège suivant :

LA VOIX DE LA NARRATRICE «Je vais vous raconter une histoire d'il y a très longtemps...»(p. 9)

LA TRES JEUNE FILLE (ne pouvant s'empêcher de raconter). Elle était fort énervée ce jour-là...

(Le père fait signe à sa fille de se taire. Elle se tait puis elle reprend.)

C'était rare pourtant qu'elle s'énerve ma mère... (p. 35)

LA TRES JEUNE FILLE Voilà l'histoire. Vous êtes contente ? (p. 51)

LA BELLE-MERE Jamais je n'aurais pu imaginer revivre un jour une histoire comme celle-là.... Aussi beau qu'un conte... ou un rêve... (p. 94)

LA TRES JEUNE FILLE Tu trouves pas qu'il $\mathrm{y}$ a comme un problème avec cette histoire?

[...] Tu penses pas des fois qu'on est en train de te raconter des histoires avec cette histoire?

[...] Je crois que des fois dans la vie on se raconte des histoires dans sa tête, on sait très bien que ce sont des histoires mais on se les raconte quand même. (p. 100)

LE TRES JEUNE PRINCE Y a quelque chose qui tournait pas rond dans cette histoire (p. 103)

SCUR LA GRANDE Vous êtes en train de vous raconter une histoire dans votre tête Majesté (107)

LA VOIX DE LA NARRATRICE Alors voilà l'histoire se termine. C'est la fin. Comme je vous l'ai dit pour commencer, je ne me rappelle plus si cette histoire est la mienne ou bien l'histoire de quelqu'un d'autre (111)

11 Pourquoi nous racontons-nous des histoires?, peut-on demander à la suite de Jérôme Bruner ${ }^{23}$ et à la lecture de ce Cendrillon: pour rêver, pour se divertir, pour raviver les schèmes des grands récits, en échappatoire ludique à l'ennui et au poids de la vie ? Lorsqu'il transpose Cendrillon pour la scène, Joël Pommerat écrit une pièce personnelle et revendique sa liberté de créateur pour, à travers la mise en scène du travail de deuil d'une orpheline, nous émouvoir tout en nous faisant rire, nous donner à penser et aussi nous enchanter lors de la représentation. Qu'il ait ou non relu les contes sources, qu'il élude ou détourne les épisodes les plus attendus importe peu et il pourrait sans doute reprendre à son compte la formule de la voix narratrice au dénouement: « je ne me rappelle plus si cette histoire est la mienne ou bien l'histoire de quelqu'un d'autre. Mais ça n'a pas d'importance ». Ainsi vont les contes, d'une réappropriation à l'autre, lorsque les auteurs font leur travail de tissage et de broderie, en intriquant ces fils entrecroisés que l'on cherche, vainement, à démêler. 


\section{NOTES}

1. Nicole Belmont, Mythe, conte et enfance. Les écritures d'Orphée et de Cendrillon. Paris, L'Harmattan, 2010, "Anthropologie du Monde Occidental». Sous la cendre - Figures de Cendrillon, anthologie établie et postfacée par Nicole Belmont et Elizabeth Lemirre, Paris, Éd. José Corti, 2007, 423 p.

2. Martial Poirson (dir.), Perrault en scène. Transpositions théâtrales de contes merveilleux, 1697-1800, Espaces 34, coll. « Théâtre du XVIII siècle », 2009, 335 p.

3. Marie Bernanoce, Conte et théâtre : quand le récit hante les dramaturgie jeunesse. Pour plus de précisions, on pourra aussi consulter le site de la BnF, URL : http://expositions.bnf.fr/contes/ gros/cendrill/

4. La textanalyse, mise en œuvre par Jean Bellemin-Noël dès 1979 (L'inconscient du texte, Paris, PUF, coll. «Ecriture ", éd. revue et augmentée en 1996) peut se définir comme une critique psychanalytique des textes littéraires.

5. Jean Bellemin-Noël, Les Contes et leurs fantasmes, Montréal, Les Editions Balzac, coll. "L'écriture indocile", 1994 (nouvelle édition revue et augmentée), p. 136. C'est lui qui souligne.

6. On retrouve ici un scénario présent dans «L'alouette qui chante et qui sautille » (KHM 88), en lien le conte de Madame Leprince de Beaumont «La Belle et la Bête » (AT 425 A), motif qui renvoie aussi bien au Roi Lear qu'à L'Âne d'or d'Apulée.

7. Jacob et Wilhelm Grimm, "Cendrillon », Contes pour les enfants et la maison, trad. N. RimassonFertin, Paris, José Corti, coll. « Merveilleux », 2009, p. 146-147.

8. Le conte des Grimm figure en effet dans le recueil de versions populaires, Les Histoires de Cendrillon racontées dans le monde, F. Morel, G. Bizouerne, Syros, 2007, dans la collection « Le tour du monde d'un conte » dirigée par Nicole Belmont.

9. Bruno Castan, La Fille aux oiseaux, Les éditions théâtrales, 2011 [1 $1^{\mathrm{e}}$ éd. 1988], p. 92 et 94.

10. Guillaume Apollinaire, La Suite de Cendrillon ou Le Rat et les six lézards, ill. N. Trovato, Passage Piétons, 2005. Jean Anouilh, « Le carrosse inutile », Fables, La Table ronde, 1967.

11. Philip Pullman, J'étais un rat, trad. A. Krief, Gallimard « Folio junior », 1999.

12. William Wegman, C. Kismaric, M. Heiferman, Cinderella, New York, Hyperion Book, 1993.

13. Suzanne Rominger, Cendrillon dépoussiérée, Paris, Retz, « Petits comédiens », 2003.

14. René Pillot, Cendres... Cendrillon, ill. Monique Czarnecki, Lille, Editions de La Fontaine, 1993.

15. Robert Walser, Cendrillon, trad. de l'allemand par Anne Longuet Marx, Paris, Mini Zoé, 2006

16. Pascale Auraix-Jonchière, "Les jeux de la reconfiguration dans Schneewittchen de Robert Walser ", dans Féeries, 9/2012, p. 137.

17. En particulier dans l'entretien avec Christian Longchamp, pour le magazine de la Monnaie, juillet 2011, URL : http://www.theatrenational.be/fr/program/276/CENDRILLON\&tab=Download 18. Publiés tous deux chez Actes Sud Papiers, coll. « Heyoka Jeunesse », en 2006 et 2009.

19. Joël Pommerat, Cendrillon, Actes Sud, «Babel», 2013, p. 12. C'est nous qui soulignons. Nous utiliserons cette édition pour toutes nos citations. Il faut toutefois signaler tout l'intérêt de l'édition du texte à destination des jeunes lecteurs, en 2012, dans la collection "Heyoka jeunesse ", chez Actes Sud-papiers, agrémentée par les remarquables illustrations en couleur de Roxane Lumeret.

20. Ibid., p. 64.

21. Charles Perrault, "Cendrillon ou la petite pantoufle de verre", Contes, Le Livre de poche, «Classique », 1990, p. 240.

22. Marc Escola, Contes de Charles Perrault, Paris, Gallimard, « Foliothèque », 2005, p. 106. 
23. Jérôme Bruner, Pourquoi nous racontons-nous des histoires? Le récit au fondement de la culture et de l'identité individuelle, trad. de l'anglais par Yves Bonin, Paris, Retz, coll. « Agora », 2002.

INDEX

Mots-clés : Cendrillon, Pommerat (Joël), conte 